\title{
Research Article \\ Dynamical Jumps in a Shape Memory Alloy Oscillator
}

\author{
H. S. Oliveira, ${ }^{1}$ A. S. de Paula, ${ }^{1}$ and M. A. Savi ${ }^{2}$ \\ ${ }^{1}$ Department of Mechanical Engineering, Universidade de Brasília, 70910-900 Brasília, DF, Brazil \\ ${ }^{2}$ Department of Mechanical Engineering, COPPE, Universidade Federal do Rio de Janeiro, P.O. Box 68.503, \\ 21941-972 Rio de Janeiro, RJ, Brazil
}

Correspondence should be addressed to A. S. de Paula; alinedepaula@unb.br

Received 26 June 2013; Accepted 10 March 2014; Published 18 August 2014

Academic Editor: Miguel Neves

Copyright (C) 2014 H. S. Oliveira et al. This is an open access article distributed under the Creative Commons Attribution License, which permits unrestricted use, distribution, and reproduction in any medium, provided the original work is properly cited.

The dynamical response of systems with shape memory alloy (SMA) elements presents a rich behavior due to their intrinsic nonlinear characteristic. SMA's nonlinear response is associated with both adaptive dissipation related to hysteretic behavior and huge changes in properties caused by phase transformations. These characteristics are attracting much technological interest in several scientific and engineering fields, varying from medical to aerospace applications. An important characteristic associated with dynamical response of SMA system is the jump phenomenon. Dynamical jumps result in abrupt changes in system behavior and its analysis is essential for a proper design of SMA systems. This paper discusses the nonlinear dynamics of a one degree of freedom SMA oscillator presenting pseudoelastic behavior and dynamical jumps. Numerical simulations show different aspects of this kind of behavior, illustrating its importance for a proper understanding of nonlinear dynamics of SMA systems.

\section{Introduction}

Shape memory alloys (SMAs) have unique thermomechanical properties responsible for their use in several applications. These remarkable properties are attracting technological interest in science and engineering fields [1-3]. In terms of applied dynamics, SMAs are being used in order to explore adaptive dissipation associated with hysteresis loop and the mechanical property changes due to phase transformation $[4,5]$. Moreover, the dynamical response of systems with SMA actuators presents a unique dynamical behavior due to their intrinsic nonlinear characteristic, presenting periodic, quasiperiodic, and chaotic responses [4, 6-8]. Recently, SMA constraints have been used for vibration reduction since it is expected that the high dissipation capacity of SMAs changes the system response producing less complex behaviors [9-11]. Another important application of SMA related to dynamical system is the adaptive tuned vibration absorbers. The main idea related to tuned vibration absorbers is the use of a secondary system connected to a main system in order to dissipate energy. In general, this idea has been used in electrical transmission lines and structural systems. Although this is very effective in tuned frequencies, it is difficult to be applied when frequency variations occur. SMA can be employed in order to confer adaptive behavior to this tuned system, allowing its application when frequency variations are expected [12].

Although SMA systems have interesting behaviors to be exploited in dynamical applications, some characteristics may be critical for practical situations. In this regard, the design of SMA system has a huge importance, being necessary in a deep investigation of the dynamical aspects of the system. Besides chaos and multistability aspects that are present in SMA dynamical systems, an important aspect is the dynamical jump. Basically, dynamical jumps are associated with nonlinear resonant response, causing abrupt changes in system behavior, introducing unstable regions on system response.

Bernardini and Rega [8] presented several aspects related to dynamical jumps in SMA systems. Basically, the authors investigated a one degree of freedom oscillator considering both isothermal and anisothermal systems. It is shown that nonregular responses occur around the jumps. In general, multicomponent harmonic-balance method, path-following technique, and Adams-Moulton algorithm were employed to investigate the system response. 
This paper revisits the dynamical jumps in a one degree of freedom SMA oscillator harmonically excited. The restitution force is provided by an SMA element described by a constitutive model with internal constraints [13]. Numerical simulations are carried out showing different aspects of the dynamical response of the system. Conclusions of this investigation establish a relationship between dynamical jumps and stress-strain relation of the SMA element.

\section{Constitutive Model}

The thermomechanical description of shape memory alloys is the objective of numerous research efforts that try to contemplate all behavior details $[1,2]$. Here, a constitutive model that is built upon Fremond's model that was previously presented in some studies $[6,13-17]$ is employed. This model considers different material properties for each phase and four macroscopic phases for the description of the SMA behavior.

Therefore, besides the total strain, $\varepsilon$, and temperature, $T$, it is necessary to define four internal variables that represent volume fraction of each macroscopic phase: $\beta_{1}$ and $\beta_{2}$, related to detwinned martensites, respectively, associated with tension and compression; $\beta_{3}$ that represents the austenitic volume fraction; and $\beta_{4}$ that represents the volume fraction of twinned martensite. Since there is a constraint based on phase coexistence, $\beta_{1}+\beta_{2}+\beta_{3}+\beta_{4}=1$, it is possible to use only three volume fractions and the thermomechanical behavior of the SMA is described by the following set of equations $[13,18]$ :

$$
\begin{aligned}
& \sigma=E \varepsilon+\left[E \alpha_{h}+\alpha\right]\left(\beta_{2}-\beta_{1}\right)-\Omega\left(T-T_{0}\right), \\
& \dot{\beta}_{1}=\frac{1}{\eta_{1}}\left\{\alpha \varepsilon+\Lambda_{1}(T)+\left(2 \alpha \alpha_{h}+E \alpha_{h}^{2}\right)\left(\beta_{2}-\beta_{1}\right)\right. \\
& \left.+\alpha_{h}\left[E \varepsilon-\Omega\left(T-T_{0}\right)\right]-\partial_{\beta_{1}} J_{\pi}\right\}+\partial_{\dot{\beta}_{1}} J_{\chi} \\
& \dot{\beta}_{2}=\frac{1}{\eta_{2}}\left\{-\alpha \varepsilon+\Lambda_{2}(T)-\left(2 \alpha \alpha_{h}+E \alpha_{h}^{2}\right)\left(\beta_{2}-\beta_{1}\right)\right. \\
& \left.-\alpha_{h}\left[E \varepsilon-\Omega\left(T-T_{0}\right)\right]-\partial_{\beta_{2}} J_{\pi}\right\}+\partial_{\dot{\beta}_{2}} J_{\chi}, \\
& \dot{\beta}_{3}=\frac{1}{\eta_{3}}\left\{-\frac{1}{2}\left(E_{A}-E_{M}\right)\left[\varepsilon+\alpha_{h}\left(\beta_{2}-\beta_{1}\right)\right]^{2}+\Lambda_{3}(T)\right. \\
& +\left(\Omega_{A}-\Omega_{M}\right)\left(T-T_{0}\right)\left[\varepsilon+\alpha_{h}\left(\beta_{2}-\beta_{1}\right)\right] \\
& \left.-\partial_{\beta_{3}} J_{\pi}\right\}+\partial_{\dot{\beta}_{3}} J_{\chi}
\end{aligned}
$$

where $\sigma$ is the stress and $E=E_{M}+\beta_{3}\left(E_{A}-E_{M}\right)$ is the elastic modulus while $\Omega=\Omega_{M}+\beta_{3}\left(\Omega_{A}-\Omega_{M}\right)$ is related to thermal expansion coefficient. Note that subscript $A$ refers to austenitic phase, while $M$ refers to martensite. Parameters $\Lambda_{1}=\Lambda_{2}=\Lambda=\Lambda(T)$ and $\Lambda_{3}=\Lambda_{3}(T)$ are associated with phase transformation stress levels. Parameter $\alpha_{h}$ defines the horizontal width of the stress-strain hysteresis loop, while $\alpha$ controls the height of the same hysteresis loop. The terms $\partial_{n} J_{\pi}$ ( $\left.n=\beta_{1}, \beta_{2}, \beta_{3}\right)$ are subdifferentials of the indicator function $J_{\pi}$ with respect to $n$. This indicator function is related to a convex set $\pi$, which provides the internal constraints related to the phase coexistence. With respect to evolution equations of volume fractions, $\eta_{1}=\eta_{2}=\eta$ and $\eta_{3}$ represent the internal dissipation related to phase transformations. Moreover $\partial_{n} J_{\chi}$ $\left(n=\beta_{1}, \beta_{2}, \beta_{3}\right)$ are subdifferentials of the indicator function $J_{\chi}$ with respect to $n$. This indicator function is associated with the convex set $\chi$, which establishes conditions for the correct description of internal subloops due to incomplete phase transformations. These subdifferentials may be replaced by Lagrange multipliers associated with the mentioned constraints [19].

Concerning parameter definitions, temperaturedependent relations are adopted for $\Lambda$ and $\Lambda_{3}$ as follows:

$$
\begin{gathered}
\Lambda= \begin{cases}-L_{0}+\frac{L}{T_{M}}\left(T-T_{M}\right), & \text { if } T>T_{M} \\
-L_{0}, & \text { if } T \leq T_{M} ;\end{cases} \\
\Lambda_{3}= \begin{cases}-L_{0}^{A}+\frac{L^{A}}{T_{M}}\left(T-T_{M}\right), & \text { if } T>T_{M} \\
-L_{0}^{A}, & \text { if } T \leq T_{M},\end{cases}
\end{gathered}
$$

where $T_{M}$ is the temperature below where the martensitic phase becomes stable. Usually, experimental tests provide information of $M_{s}$ and $M_{f}$, temperatures of the start and finish of the martensitic formation. This model uses only one temperature that could be an average value or, alternatively, the $M_{f}$ value. Moreover, $L_{0}, L, L_{0}^{A}$, and $L^{A}$ are parameters related to critical stress for phase transformation.

In order to describe the characteristics of phase transformation kinetics, different values of $\eta$ and $\eta_{3}$ might be considered during loading, $\eta^{L}$ and $\eta_{3}^{L}$, and unloading processes, $\eta^{U}$ and $\eta_{3}^{U}$. For more details about the constitutive model, see $[13,16]$. All constitutive parameters can be matched from stress-strain tests.

As it is well known, SMA devices demonstrate timedependent characteristics which means that their thermomechanical response depends on the loading rate; see, for example, $[20,21]$. The proper modeling of this time dependency can be performed by considering the thermomechanical coupling terms in the energy equation. Reference [22] discusses the thermomechanical coupling and rate dependency in SMAs.

The considered constitutive model has viscous characteristic that allows the description of the thermomechanical coupling avoiding the integration of the energy equation, presenting useful results [15]. Reference [22] explores the same idea showing the difference between a viscous model and a rate-independent model with thermomechanical coupling. Both models have the ability to describe pseudoelastic behavior in SMA wires. This time-dependent aspect can be controlled by the proper choice of model parameters.

\section{Single Degree of Freedom Shape Memory Oscillator}

The dynamical behavior of SMAs is analyzed by considering a single degree of freedom oscillator (1DOF) with two different forcing possibilities. Initially, a harmonic base 


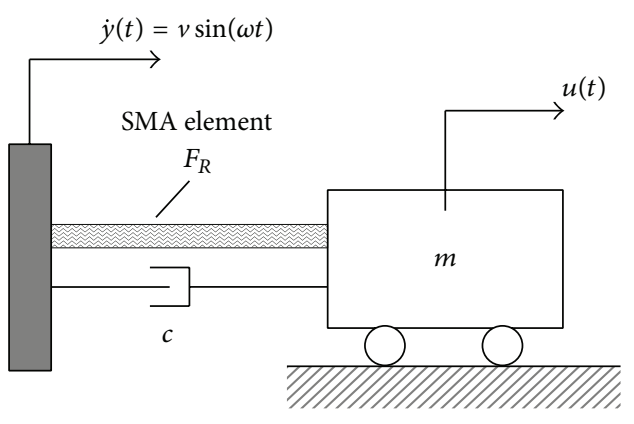

(a)

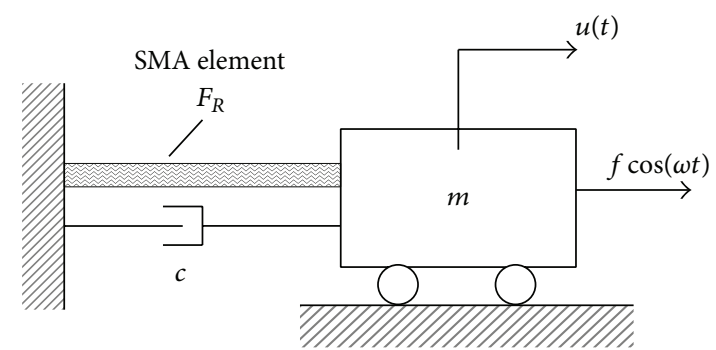

(b)

FIGURE 1: Single degree of freedom oscillator: (a) harmonic base excitation and (b) harmonic force applied to the mass.

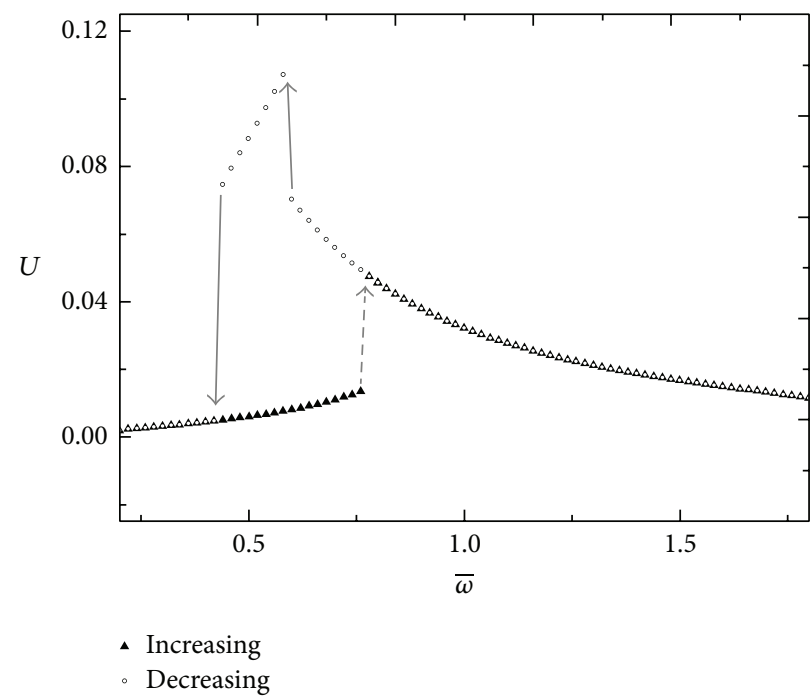

FIGURE 2: Maximum system amplitude with $v=0.025 \mathrm{~m} / \mathrm{s}$ and a sine sweep of the forcing frequency.

excitation with prescribed velocity $\dot{y}(t)=v \sin (\omega t)$, as shown in Figure 1(a), is considered. Then, a harmonic excitation $F_{0}(t)=f \cos (\omega t)$ applied directly to the oscillator mass, as presented in Figure 1(b), is analyzed. The motivation of studying these two situations is the development of SMA vibration absorbers and isolators. The oscillator presented in Figure 1(a), for example, can be used to analyze classical dynamical absorbers, which are attached to a primary system that presents a harmonic response that must have its response amplitude reduced. The oscillator of Figure 1(b), on the other hand, can be used to analyze vibration reduction of systems harmonically excited, which occurs in rotating machines due to unavoidable unbalance. Therefore, the dynamical analysis of these systems is an important start for the design of SMA devices.

The SMA oscillator consists of a mass $m$ attached to a shape memory element of length $l$ and cross-sectional area $A$ and restitution force $F_{R}$ (Figure 1). A linear viscous damper, characterized by a viscous coefficient $c$, is also considered in order to represent dissipations different from the dissipation associated with the SMA element.
The equation of motion of this oscillator may be formulated by considering the balance of forces acting on the mass as follows:

$$
m \ddot{u}+c \dot{u}+F_{R}=F_{0} \cos (\omega t),
$$

where $F_{R}=\sigma A, u=(x-y), F_{0}=-m v \omega$ in the case of base excitation and $F_{0}=f$ when the force is applied directly to the oscillator. The restitution force of the oscillator is provided by an SMA element described by the constitutive equations presented in the previous section [13]. Therefore, the following equation of motion is obtained [4]:

$$
\begin{gathered}
m \ddot{u}+c \dot{u}+\frac{E A}{l} u+\left(A \alpha+E A \alpha_{h}\right)\left(\beta_{2}-\beta_{1}\right) \\
-\Omega A\left(T-T_{0}\right)=F_{0} \cos (\omega t),
\end{gathered}
$$

where volume fractions of evolution $\beta_{1}$ and $\beta_{2}$ are described by the constitutive model presented in the preceding section 


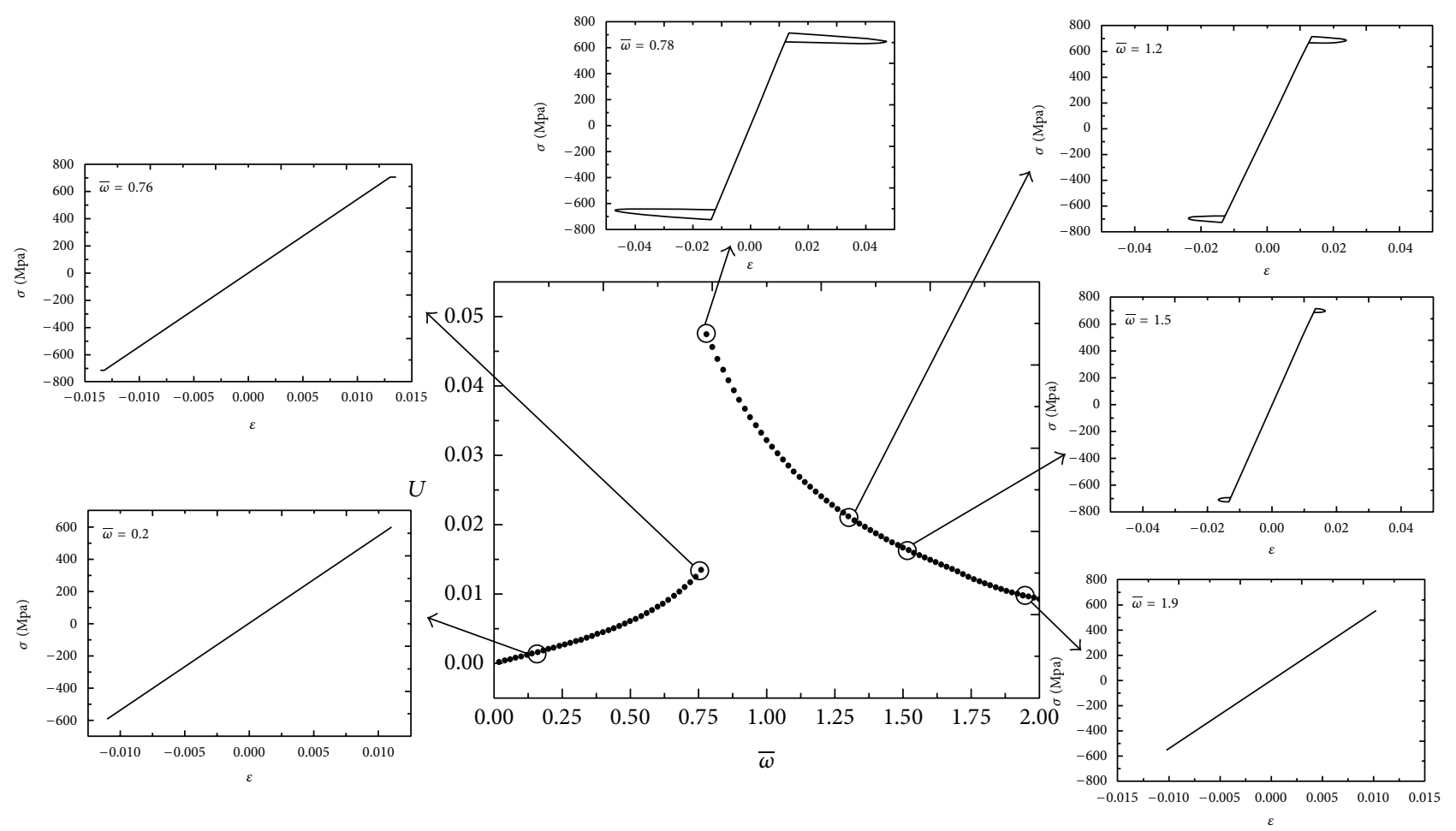

FIGURE 3: Up-sweep jumps with $v=0.025 \mathrm{~m} / \mathrm{s}$ : comparison between frequency response and stress-strain curves.
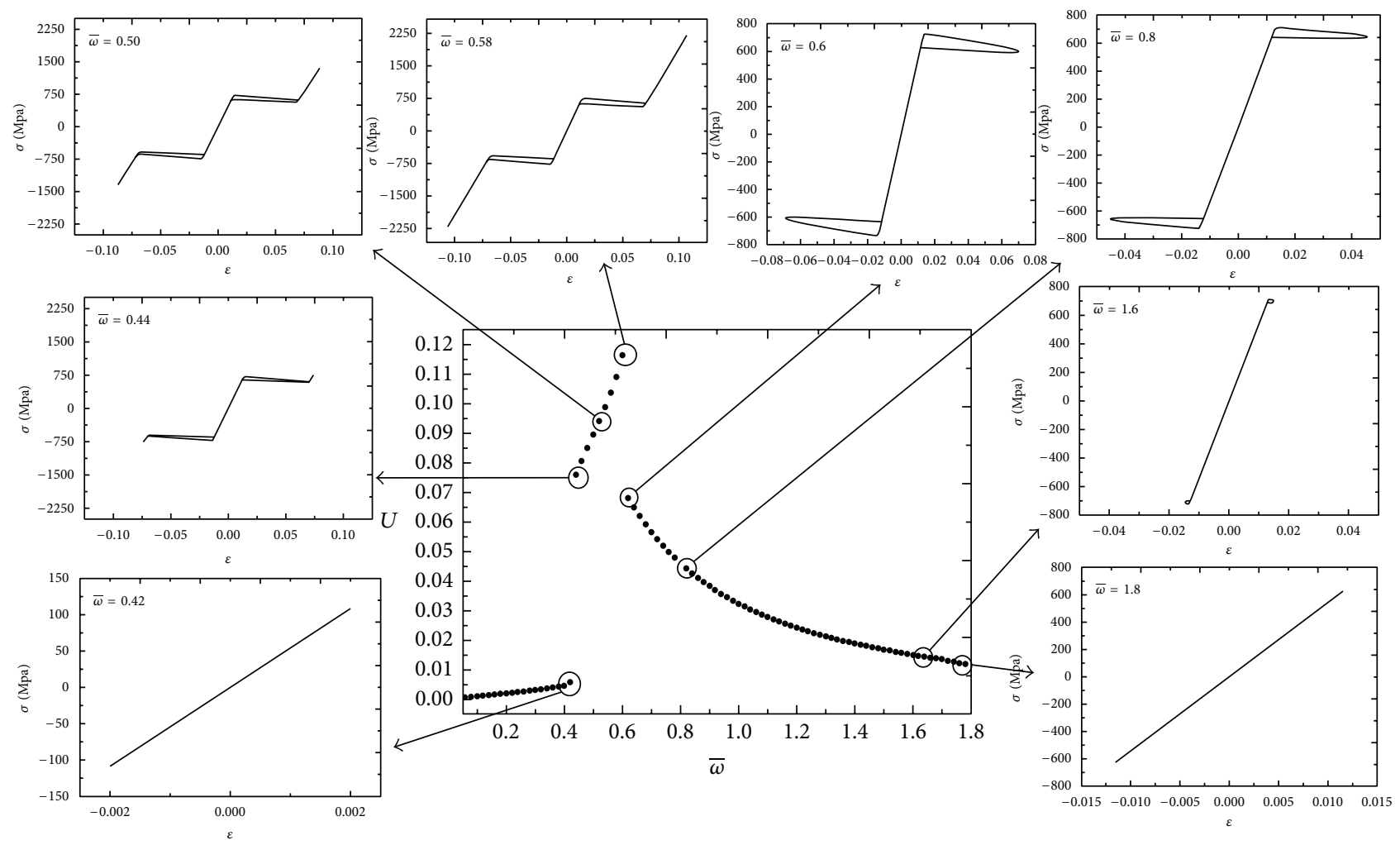

FIGURE 4: Down-sweep jumps with $v=0.025 \mathrm{~m} / \mathrm{s}$ : comparison between frequency response and stress-strain curves. 


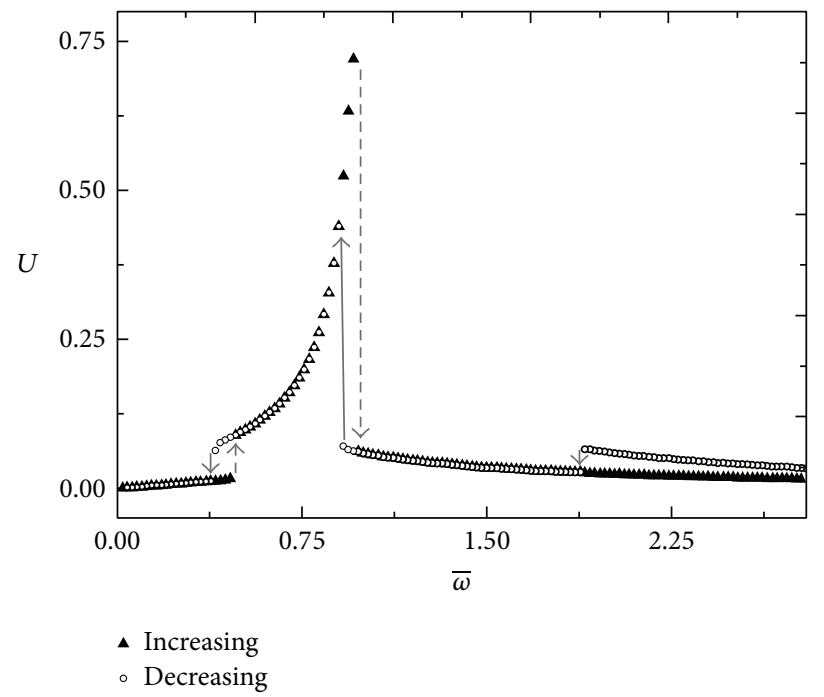

FIgURE 5: Maximum system amplitude with $v=0.075 \mathrm{~m} / \mathrm{s}$ and increasing the forcing frequency.
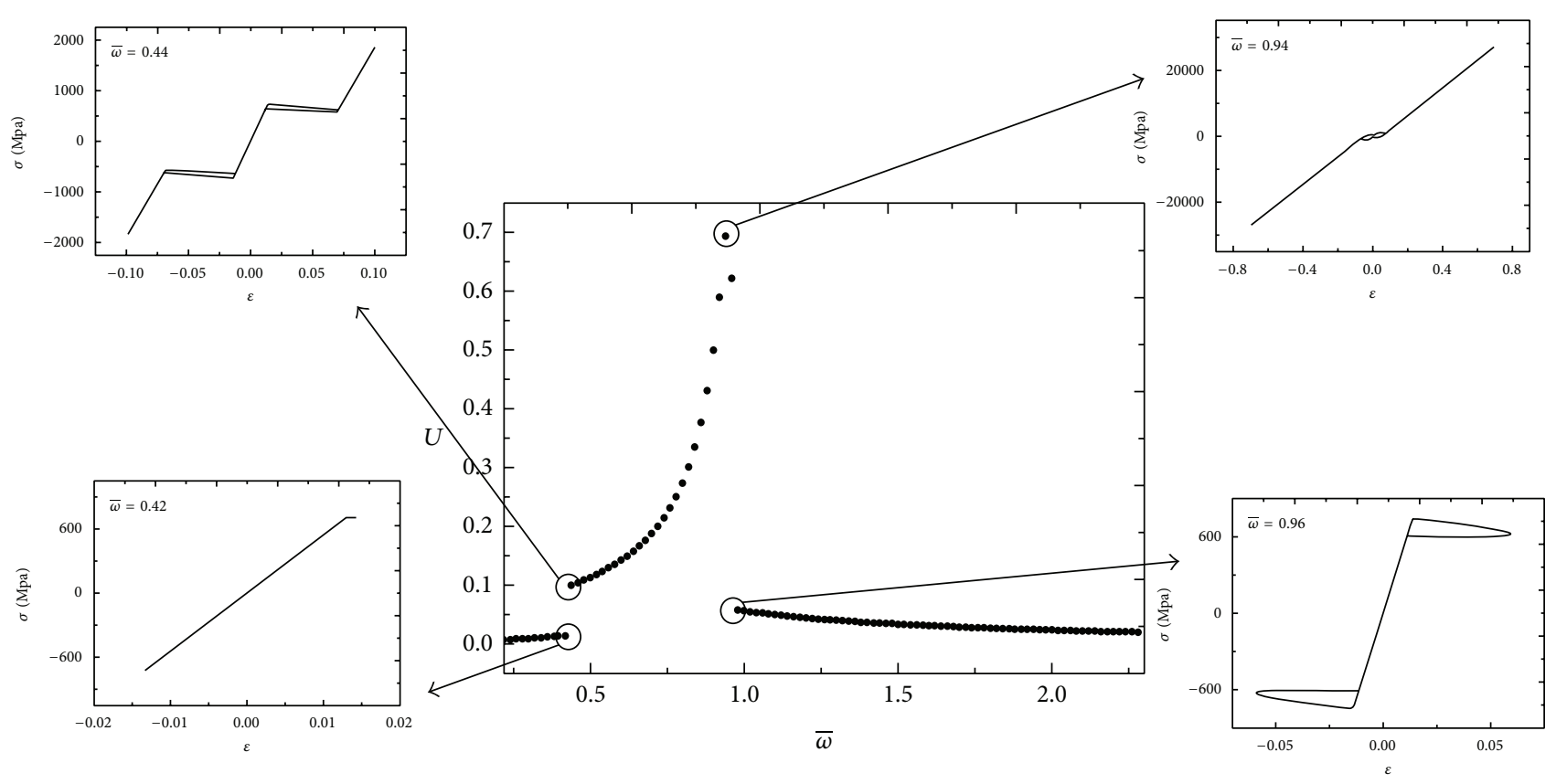

FIGURE 6: Up-sweep jumps with $v=0.075 \mathrm{~m} / \mathrm{s}$ : comparison between frequency response and stress-strain curves.

and $\varepsilon=u / l$. In order to obtain a dimensionless equation of motion, the system's parameters are defined as follows:

$$
\begin{gathered}
\bar{\Omega}=\frac{\Omega_{R} A T_{R}}{m l \omega_{0}^{2}}=\frac{\Omega_{R} T_{R}}{E_{R}} ; \quad \bar{\alpha}=\frac{\alpha A}{m l \omega_{0}^{2}}=\frac{\alpha}{E_{R}} ; \\
\bar{\alpha}_{h}=\frac{\alpha_{h} E_{R} A}{m l \omega_{0}^{2}} ; \quad \omega_{0}^{2}=\frac{E_{R} A}{m l} ; \\
\xi=\frac{c}{c \omega_{0}} ; \quad \mu_{E}=\frac{E}{E_{R}} ; \\
V=\frac{v}{\omega_{0} l} ; \quad \mu_{\Omega}=\frac{\Omega}{\Omega_{R}} ; \quad \bar{\omega}=\frac{\omega}{\omega_{0}} .
\end{gathered}
$$

Note that dimensionless parameters and variables are defined considering some reference values for temperaturedependent parameters. This is done by assuming a reference temperature, $T_{R}$, where these parameters are evaluated. Therefore, parameters with subscript $R$ are evaluated at this reference temperature. These definitions allow one to define the following dimensionless variables, respectively, related to mass displacement $(U)$, temperature $(\theta)$, and time $(\tau)$ :$$
U=\frac{u}{l} ; \quad \theta=\frac{T}{T_{R}} ; \quad \tau=\omega_{0} t
$$ 

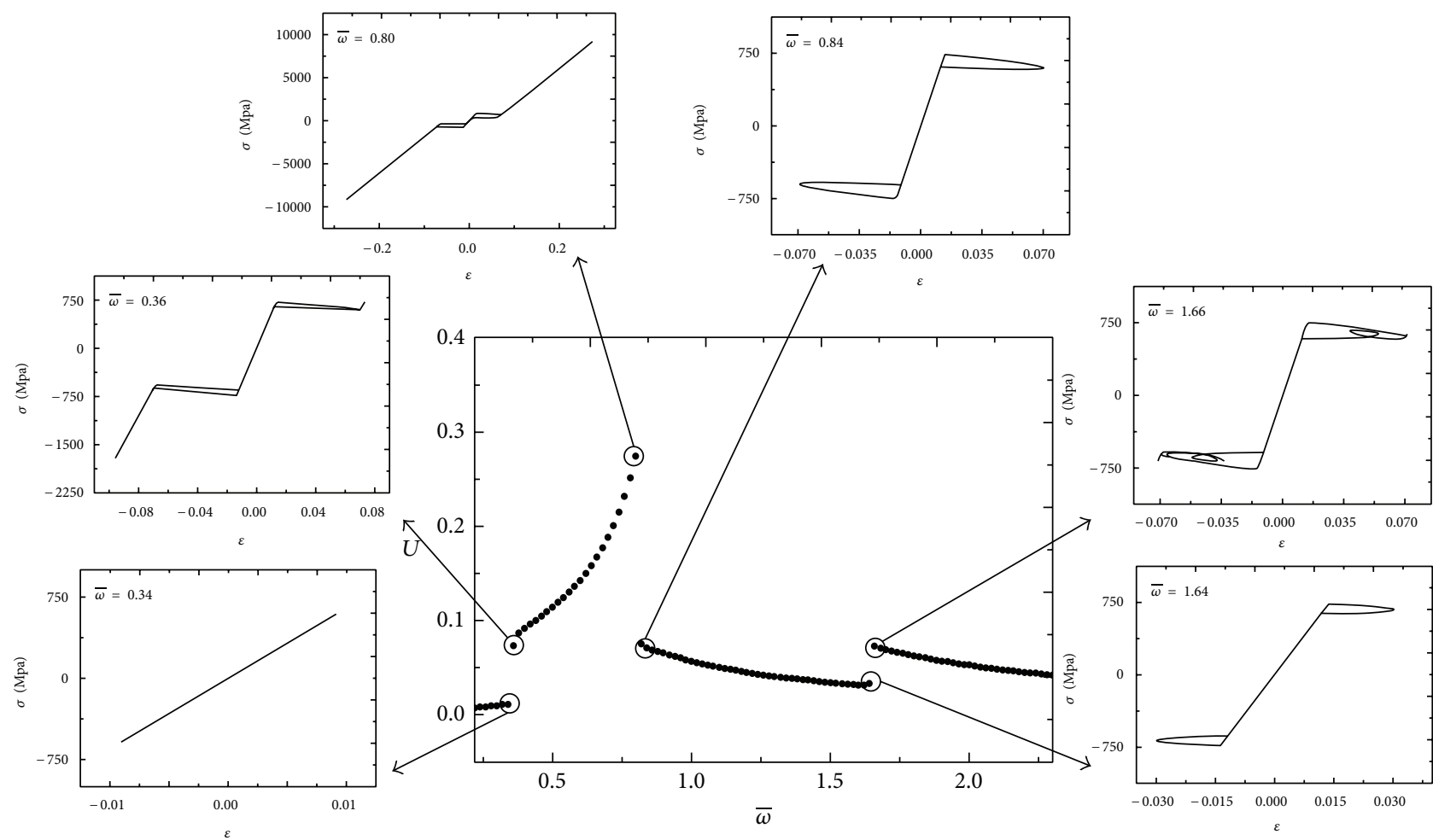

FIGURE 7: Down-sweep jumps with $v=0.075 \mathrm{~m} / \mathrm{s}$ : comparison between frequency response and stress-strain curves.

Therefore, the dimensionless equation of motion has the following form:

$$
\begin{aligned}
& U^{\prime \prime}+\xi U^{\prime}+\mu_{E} U+\left(\bar{\alpha}+\mu_{E} \bar{\alpha}_{h}\right)\left(\beta_{2}-\beta_{1}\right) \\
& -\mu_{\Omega} \bar{\Omega}\left(\theta-\theta_{0}\right)=\delta \cos (\bar{\omega} t), \\
& \beta_{1}^{\prime}=\frac{1}{\bar{\eta}_{1}}\left\{\alpha \varepsilon+\Lambda_{1}(T)+\left(2 \alpha \alpha_{h}+E \alpha_{h}^{2}\right)\left(\beta_{2}-\beta_{1}\right)\right. \\
& \left.+\alpha_{h}\left[E \varepsilon-\Omega\left(T-T_{0}\right)\right]-\partial_{\beta_{1}} J_{\pi}\right\}+\partial_{\dot{\beta}_{1}} J_{\chi}, \\
& \beta_{2}^{\prime}=\frac{1}{\bar{\eta}_{2}}\left\{-\alpha \varepsilon+\Lambda_{2}(T)-\left(2 \alpha \alpha_{h}+E \alpha_{h}^{2}\right)\left(\beta_{2}-\beta_{1}\right)\right. \\
& \left.-\alpha_{h}\left[E \varepsilon-\Omega\left(T-T_{0}\right)\right]-\partial_{\beta_{2}} J_{\pi}\right\}+\partial_{\dot{\beta}_{2}} J_{\chi}, \\
& \beta_{3}^{\prime}=\frac{1}{\bar{\eta}_{3}}\left\{-\frac{1}{2}\left(E_{A}-E_{M}\right)\left[\varepsilon+\alpha_{h}\left(\beta_{2}-\beta_{1}\right)\right]^{2}+\Lambda_{3}(T)\right. \\
& +\left(\Omega_{A}-\Omega_{M}\right)\left(T-T_{0}\right)\left[\varepsilon+\alpha_{h}\left(\beta_{2}-\beta_{1}\right)\right] \\
& \left.-\partial_{\beta_{3}} J_{\pi}\right\}+\partial_{\dot{\beta}_{3}},
\end{aligned}
$$

where derivatives with respect to dimensionless time are represented by ()$^{\prime}=d() / d \tau, \bar{\eta}_{i}=\eta_{i} / \omega_{0}$, and $\delta=$ $-m v \omega / m l \omega_{0}^{2}=-m v \omega / E_{R} A$ in the case of base excitation and $\delta=f / m l \omega_{0}^{2}=f / E_{R} A$ when the force is applied directly to the oscillator.

\section{Numerical Simulation}

This section presents a numerical investigation of the 1DOF SMA oscillator. In order to deal with nonlinearities of the SMA oscillator equations of motion, an iterative procedure based on the operator split technique [23] is employed. Under this assumption, the fourth-order Runge-Kutta method is used together with the projection algorithm proposed in [6] to solve the constitutive equations. The solution of the constitutive equations also employs the operator split technique together with an implicit Euler method. The calculation of $\beta_{n}$ $(n=1,2,3)$ considers that the evolution equations are solved in a decoupled way. At first, the equations (except for the subdifferentials) are solved using an iterative implicit Euler method. If the estimated results obtained for $\beta_{n}$ do not satisfy the imposed constraints, an orthogonal projection algorithm pulls their value to the nearest point on the domain's surface [19]. On the other hand, the numerical integration of the dynamical system uses the classical Runge-Kutta method.

Parameters used in the numerical simulations, presented in Table 1, are the same used by Savi et al. [4]. These parameters are obtained by calibrating the model to experimental results of a NiTi alloy. Simulations are carried out at a temperature of $372 \mathrm{~K}$, where only austenitic phase is stable at stress-free state.

The dynamical analysis of the SMA oscillator is mainly focused on the investigation of the dynamical jumps. The idea is to investigate numerical simulations related to sine-sweep tests of the forcing frequency. In this test, system response is investigated when the forcing frequency is increased 
TABLE 1: SMA parameters.

\begin{tabular}{lccccccc}
\hline$E_{R}(\mathrm{GPa})$ & $E_{M}(\mathrm{GPa})$ & $\alpha(\mathrm{MPa})$ & $\alpha_{h}$ & $L_{0}(\mathrm{MPa})$ & $L(\mathrm{MPa})$ & $L_{0}{ }^{A}(\mathrm{MPa})$ & $L^{A}(\mathrm{MPa})$ \\
\hline 54 & 42 & 330 & 0.048 & 0.015 & 41.5 & 0.63 & 185 \\
\hline$\Omega_{A}(\mathrm{MPa} / \mathrm{K})$ & $\Omega_{M}(\mathrm{MPa} / \mathrm{K})$ & $T_{M}(\mathrm{~K})$ & $\eta^{L}(\mathrm{Mpa} \cdot \mathrm{s})$ & $\eta^{U}(\mathrm{Mpa} \cdot \mathrm{s})$ & $\eta_{3}{ }^{L}(\mathrm{Mpa} \cdot \mathrm{s})$ & $\eta_{3}{ }^{U}(\mathrm{Mpa} \cdot \mathrm{s})$ & \\
\hline 0.74 & 0.17 & 291.4 & 1.0 & 2.7 & 1.0 & 2.7 & \\
\hline
\end{tabular}

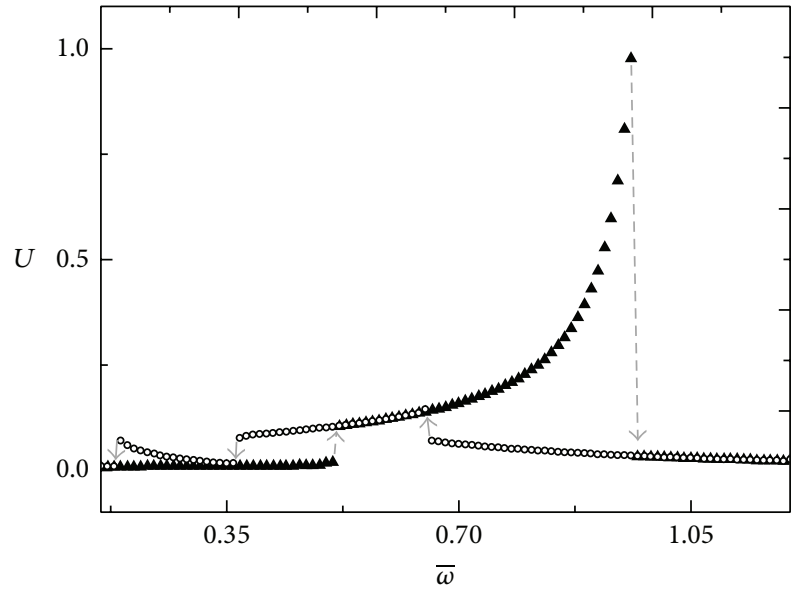

A Increasing

- Decreasing

FIGURE 8: Maximum system amplitude with $\delta=0.012$ and increasing the forcing frequency.

(up-sweep) or decreased (down-sweep) by some increment value. For each forcing frequency, the system is numerically integrated and the maximum amplitude in steady state is considered. When the frequency is changed, the last state at the previous forcing frequency is considered as the initial condition for the new frequency. This brute-force procedure is enough to identify dynamical jumps although it does not allow the identification of unstable paths related to the system response.

4.1. Harmonic Base Excitation. Initially, the SMA oscillator subjected to base excitation is of concern with $\xi=0.05$. Figure 2 presents the system response considering the sinesweep test of the frequency for $v=0.025 \mathrm{~m} / \mathrm{s}$. It is noticeable that there is one jump during the up-sweep while the downsweep presents two jumps. This behavior is caused by system nonlinearities, specifically, hysteretic behavior. During the up-sweep, dynamical jump occurs around $\bar{\omega}=0.77$. During the down-sweep, the first one occurs around $\bar{\omega}=0.59$ and the second one occurs around $\bar{\omega}=0.43$. Bernardini and Rega [8] identified similar situations with different approaches and a different constitutive model.

In order to have a better understanding about the dynamical jumps, an investigation is carried out establishing a relation between jumps and phase transformations. Figure 3 shows up-sweep frequency response curves highlighting the stress-strain curves for different forcing frequencies. It can be observed that the jump occurs when phase transformations start to occur. Therefore, linear stress-strain curve is changed to a hysteretic behavior associated with incomplete phase transformations. Note that, for frequencies smaller than $\bar{\omega}=$ 0.76 , the system has a linear behavior. By slightly increasing the forcing frequency, the system presents a hysteretic behavior. The hysteresis loop causes a significant increase of the strain, which produces a dynamical jump. By continuing to increase the forcing frequency the hysteresis loop starts to become smaller until it disappears and the system presents a linear behavior again.

Figure 4 shows the same analysis related to down-sweep test. The first jump occurs when the system changes from a situation where incomplete phase transformations are in course to a new one where complete phase transformations are occurring. This is noticeable by observing the presence of the elastic response related to martensitic phase in the stress-strain curve. Note that a very slight change around $\bar{\omega}=0.6$ changes the stress level promoting complete phase transformations, presenting an elastic response on the martensitic phase. The second jump, on the other hand, occurs where phase transformation is not occurring anymore. Note that, near $\bar{\omega}=0.44$, phase transformations are not occurring anymore causing an abrupt change from full loop to linear case.

It should be pointed out that, for the analyzed range, Figures 2-4 present a linear evolution of the frequency response when system has a linear response, without reaching the hysteretic behavior. Afterward, when the system presents a hysteretic response related to incomplete phase transformation, the response is associated with a nonlinear curve. The transition between one behavior and the other modifies the frequency response.

Now, the same analysis is carried out with a higher velocity $v=0.075 \mathrm{~m} / \mathrm{s}$. Figure 5 shows sine-sweep maximum amplitudes of the SMA oscillator. Under this new condition, the system presents two jumps during the up-sweep while the down-sweep presents three jumps. During the up-sweep (Figure 6), the first jump occurs when the response shifts from a linear behavior to a nonlinear behavior with the complete hysteresis loop. The second jump is related to the transition from the complete phase transformations to incomplete ones. Although the jumps are being caused by changes in stress-strain curves, it should be highlighted that the first jump, where the system goes from a complete hysteresis loop to a linear behavior, is different from the previous case.

Figure 7 establishes the comparison between jumps and phase transformation for the down-sweep case. The first one occurs around $\bar{\omega}=1.66$, where the maximum amplitude decreases slightly. It is noticeable that incomplete phase transformation changes from a situation where internal 


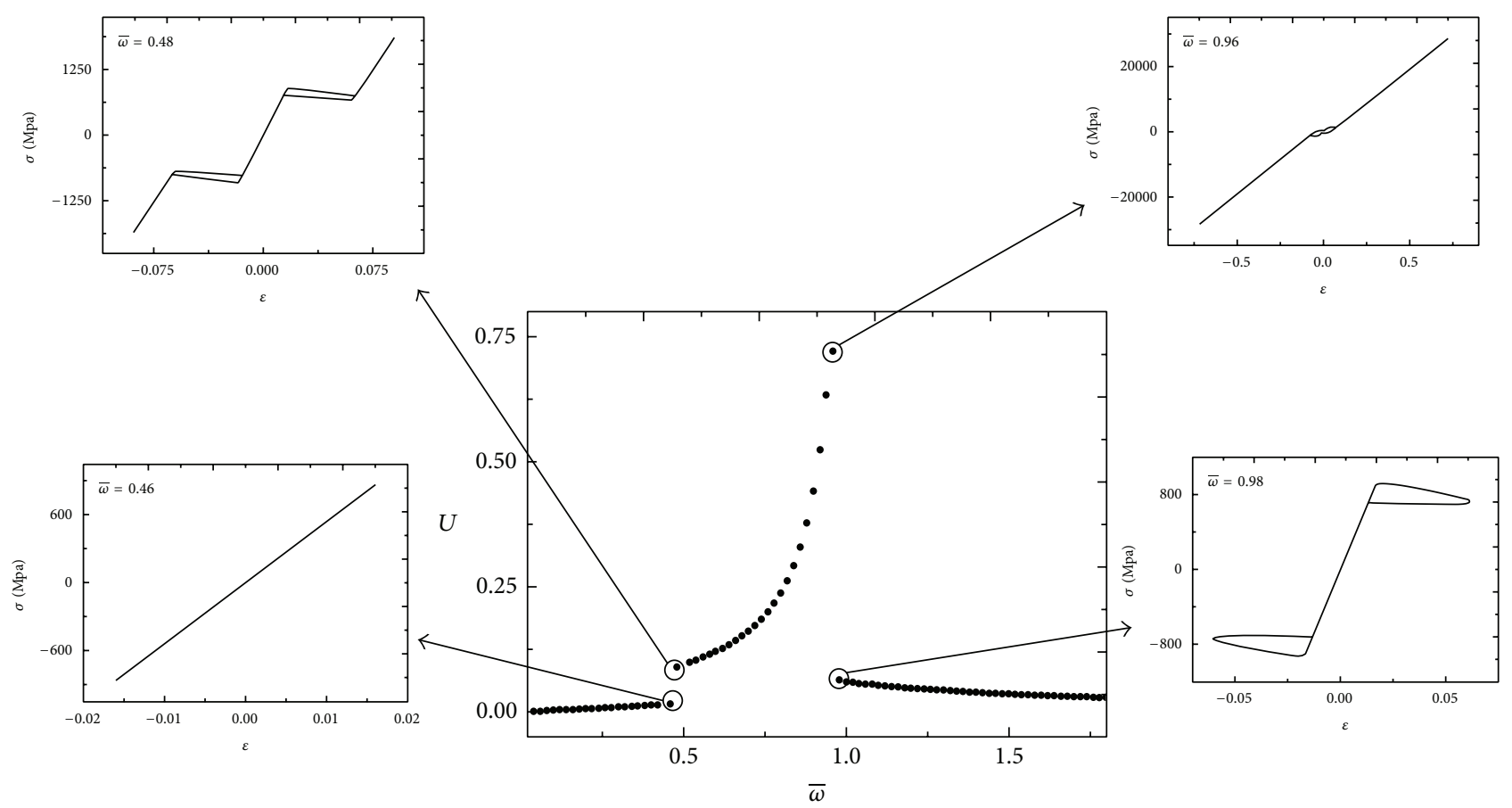

FIGURE 9: Up-sweep jumps with $\delta=0.012$ : comparison between frequency response and stress-strain curves.

subloops appear in stress-strain curves to a behavior without internal subloops. The second jump takes place at $\bar{\omega}=0.8$, where no transition in stress-strain curve is observed. The last dynamical jump occurs when the material shifts from nonlinear behavior related to an incomplete hysteresis loop to a linear behavior.

It is important to mention that when a dynamical jump occurs, it is possible to have the transition between the behaviors in stress-strain diagram immediately before the dynamical jump. In Figure 7, for example, between the stressstrain diagrams presented at $\bar{\omega}=0.84$ (incomplete hysteresis loop) and $\bar{\omega}=0.80$ (complete hysteresis loop), there is a complete hysteresis loop at $\bar{\omega}=0.82$, where the response amplitude is still low. This happens because the frequency variation is very small and the amount of the energy that increases from $\bar{\omega}=0.84$ to $\bar{\omega}=0.92$ is only sufficient to complete the hysteresis loop. After the loop is complete, even a very small increase of energy is enough to lead to the dynamical jump. Probably, this behavior occurs only in numerical simulations where a quasistatic variation of the forcing frequency is possible. In experimental tests, this subtle transition is probably not possible to be reached and only the response after the jump is captured.

4.2. Harmonic Force Applied to the Mass. At this point, the case where harmonic excitation is applied directly to SMA oscillator mass is analyzed, with $\xi=0.01$. The main idea is to establish a qualitative comparison between the responses of both oscillators. The same order of magnitude for the forcing parameters and viscous damping coefficient is considered. Nevertheless, the forcing amplitude depends on the forcing frequency in the case of base excitation while in the other case the forcing amplitude is constant. Therefore, the qualitative comparison tries to show different possibilities when each forcing condition is considered.

Figure 8 presents system response considering the sinesweep of the frequency with $\delta=0.012$. During the upsweep test two jumps are observed while in down-sweep three jumps are observed. In the up-sweep, presented in Figure 9, the first jump occurs when the response shifts from an incomplete hysteresis to a complete one. This jump consists in a different situation compared with the equivalent base excitation cases, where the transition related to the first dynamical jumps is from linear behavior to nonlinear behavior with complete hysteresis loop. The second jump is related to the inverse transition, from complete to incomplete loop.

Figure 10 establishes the comparison between jumps and phase transformation for the down-sweep case with $\delta=$ 0.012 . The first dynamical jump, which happens around $\bar{\omega}=$ 0.65 , is similar to the first jump of the down-sweep with base excitation with $v=0.025 \mathrm{~m} / \mathrm{s}$, when the transition from incomplete to complete hysteresis loop occurs. The second one occurs around $\bar{\omega}=0.36$ in a transition from complete to incomplete hysteresis loop. This situation is also verified in the previous cases. The last dynamical jump, around $\bar{\omega}=0.18$, occurs when the system goes from incomplete hysteresis loop to linear behavior. This jump, with a transition from incomplete hysteresis loop to linear behavior, consists in a different situation compared with the equivalent base excitation case.

It is important to mention that all regions related to dynamical jumps are associated with coexistence of attractors, due to the different possibilities of response when 


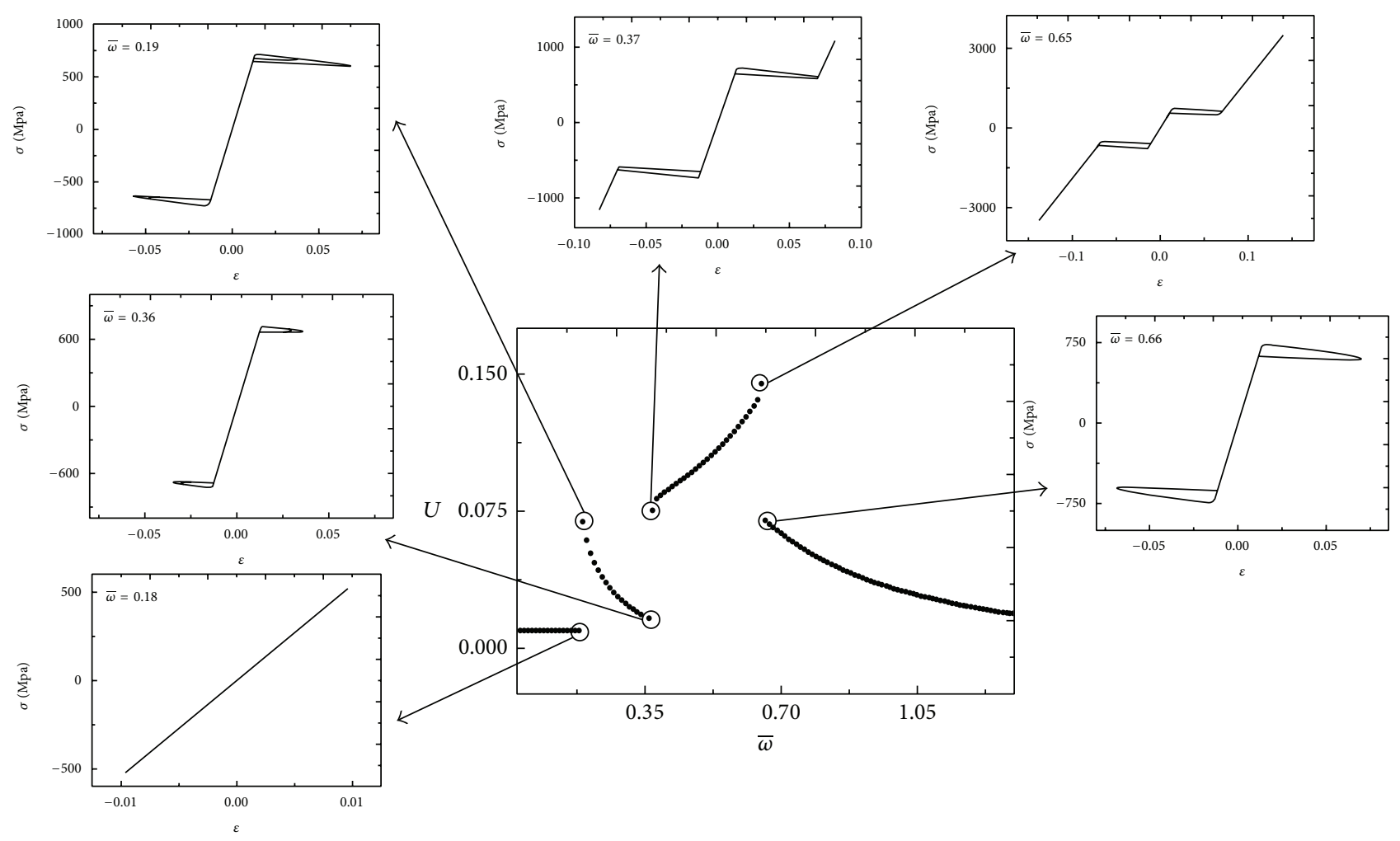

FIgURE 10: Up-sweep jumps with $\delta=0.012$ : comparison between frequency response and stress-strain curves.

increasing and decreasing the forcing frequency. This coexistence is associated with dynamical instability since small perturbations can cause the system to jump from one behavior to the other which is completely different. This kind of behavior is critical to system response and is important to be investigated.

\section{Conclusions}

This paper deals with the analysis of dynamical jumps in a shape memory alloy system. Basically, a nonlinear one degree of freedom oscillator with harmonic excitation is of concern. Two different situations are treated: base excitation and excitation applied directly to the oscillator mass. This system presents several dynamical jumps and their occurrence depends not only on the forcing amplitude-which depends on the velocity in the case of base excitation-but also on the way the forcing frequency is modified. Numerical simulations of the sine-sweep test are carried out showing that downsweep causes more dynamical jumps than up-sweep. These jumps can be explained by phase transformation arguments, observing stress-strain curves. In this regard, three possible behaviors can be observed: linear behavior, minor hysteresis loops related to incomplete phase transformation, and major hysteresis loops related to complete phase transformation. The transition between these three behaviors leads to changes in system response, which can be a dynamical jump or a modification in the frequency response of the system. It is observed that the transition between linear and incomplete hysteresis loops can lead to dynamical jumps or modifications in the frequency response of the system. All other transitions lead to dynamical jumps.

\section{Conflict of Interests}

The authors declare that there is no conflict of interests regarding the publication of this paper.

\section{Acknowledgments}

The authors would like to acknowledge the support of the Brazilian Research Agencies CNPq, CAPES, and FAPERJ and through the INCT-EIE (National Institute of Science and Technology-Smart Structures in Engineering) the CNPq and FAPEMIG. The Air Force Office of Scientific Research (AFOSR) is also acknowledged.

\section{References}

[1] D. C. Lagoudas, Shape Memory Alloys: Modeling and Engineering Applications, Springer, New York, NY, USA, 2008.

[2] A. Paiva and M. A. Savi, "An overview of constitutive models for shape memory alloys," Mathematical Problems in Engineering, vol. 2006, Article ID 56876, 30 pages, 2006.

[3] L. G. Machado and M. A. Savi, "Medical applications of shape memory alloys," Brazilian Journal of Medical and Biological Research, vol. 36, no. 6, pp. 683-691, 2003.

[4] M. A. Savi, M. A. N. Sá, A. Paiva, and P. M. C. L. Pacheco, "Tensile-compressive asymmetry influence on shape memory 
alloy system dynamics," Chaos, Solitons and Fractals, vol. 36, no. 4, pp. 828-842, 2008.

[5] J. Van Humbeeck, "Damping capacity of thermoelastic martensite in shape memory alloys," Journal of Alloys and Compounds, vol. 355, no. 1-2, pp. 58-64, 2003.

[6] M. A. Savi and P. M. C. L. Pacheco, "Chaos and hyperchaos in shape memory systems," International Journal of Bifurcation and Chaos, vol. 12, no. 3, pp. 645-657, 2002.

[7] L. G. Machado, M. A. Savi, and P. M. C. L. Pacheco, "Nonlinear dynamics and chaos in coupled shape memory oscillators," International Journal of Solids and Structures, vol. 40, no. 19, pp. 5139-5156, 2003.

[8] D. Bernardini and G. Rega, "Thermomechanical modelling, nonlinear dynamics and chaos in shape memory oscillators," Mathematical and Computer Modelling of Dynamical Systems, vol. 11, no. 3, pp. 291-314, 2005.

[9] E. Sitnikova, E. Pavlovskaia, and M. Wiercigroch, "Dynamics of an impact oscillator with SMA constraint," The European Physical Journal Special Topics, vol. 165, no. 1, pp. 229-238, 2008.

[10] E. Sitnikova, E. Pavlovskaia, M. Wiercigroch, and M. A. Savi, "Vibration reduction of the impact system by an SMA restraint: numerical studies," International Journal of NonLinear Mechanics, vol. 45, no. 9, pp. 837-849, 2010.

[11] B. C. dos Santos and M. A. Savi, "Nonlinear dynamics of a nonsmooth shape memory alloy oscillator," Chaos, Solitons and Fractals, vol. 40, no. 1, pp. 197-209, 2009.

[12] M. A. Savi, A. S. de Paula, and D. C. Lagoudas, "Numerical investigation of an adaptive vibration absorber using shape memory alloys," Journal of Intelligent Material Systems and Structures, vol. 22, no. 1, pp. 67-80, 2011.

[13] A. Paiva, M. A. Savi, A. M. B. Braga, and P. M. C. L. Pacheco, "A constitutive model for shape memory alloys considering tensile-compressive asymmetry and plasticity," International Journal of Solids and Structures, vol. 42, no. 11-12, pp. 3439-3457, 2005.

[14] S. A. Oliveira, M. A. Savi, and A. L. Kalamkarov, "A threedimensional constitutive model for shape memory alloys," Archive of Applied Mechanics, vol. 80, no. 10, pp. 1163-1175, 2010.

[15] R. A. A. Aguiar, M. A. Savi, and P. M. C. L. Pacheco, "Experimental and numerical investigations of shape memory alloy helical springs," Smart Materials and Structures, vol. 19, no. 2, Article ID 025008, 9 pages, 2010.

[16] M. A. Savi and A. Paiva, "Describing internal subloops due to incomplete phase transformations in shape memory alloys," Archive of Applied Mechanics, vol. 74, no. 9, pp. 637-647, 2005.

[17] A. P. Baêta-Neves, M. A. Savi, and P. M. C. L. Pacheco, "On the Fremond's constitutive model for shape memory alloys," Mechanics Research Communications, vol. 31, no. 6, pp. 677-688, 2004.

[18] J. Lemaitre and J. L. Charboche, Mechanics of Solid Materials, Cambridge University Press, Cambridge, UK, 1990.

[19] M. A. Savi, A. Paiva, A. P. Baêta-Neves, and P. M. C. L. Pacheco, "Phenomenological modeling and numerical simulation of shape memory alloys: a thermo-plastic-phase transformation coupled model," Journal of Intelligent Material Systems and Structures, vol. 13, no. 5, pp. 261-273, 2002.

[20] J. A. Shaw and S. Kyriakides, "Thermomechanical aspects of NiTi," Journal of the Mechanics and Physics of Solids, vol. 43, no. 8, pp. 1243-1281, 1995.

[21] S. H. Yoon and D. J. Yeo, "Experimental investigation of thermomechanical behaviors in Ni-Ti shape memory alloy," Journal of
Intelligent Material Systems and Structures, vol. 19, no. 3, pp. $283-$ 289, 2008.

[22] P. C. C. Monteiro Jr., M. A. Savi, T. A. Netto, and P. M. C. L. Pacheco, "A phenomenological description of the thermomechanical coupling and the rate-dependent behavior of shape memory alloys," Journal of Intelligent Material Systems and Structures, vol. 20, no. 14, pp. 1675-1687, 2009.

[23] M. Ortiz, P. M. Pinsky, and R. L. Taylor, "Operator split methods for the numerical solution of the elastoplastic dynamic problem," Computer Methods in Applied Mechanics and Engineering, vol. 39, no. 2, pp. 137-157, 1983. 

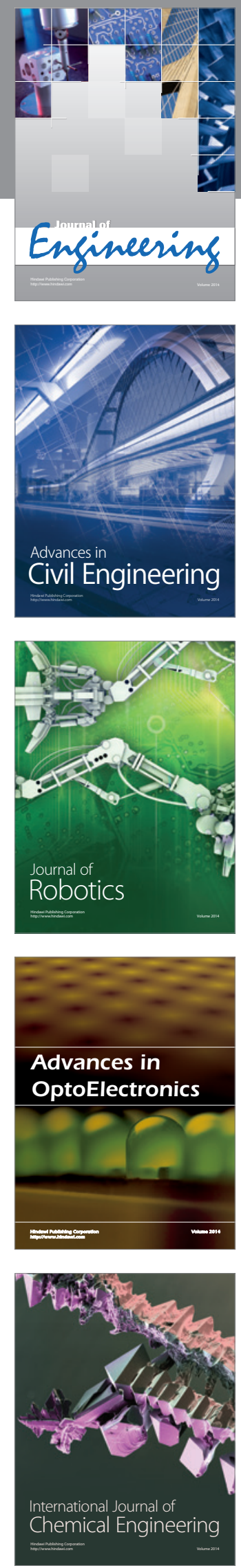

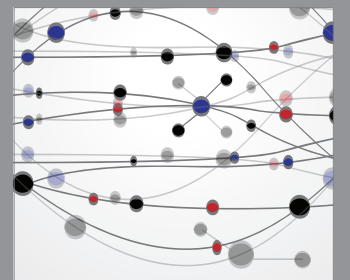

The Scientific World Journal
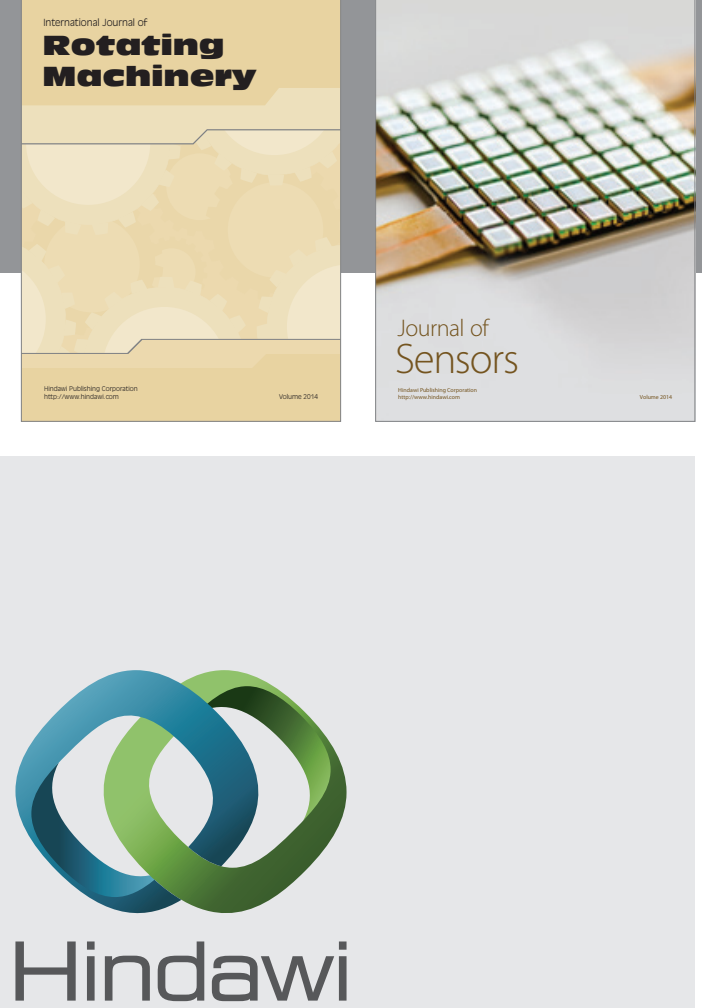

Submit your manuscripts at http://www.hindawi.com
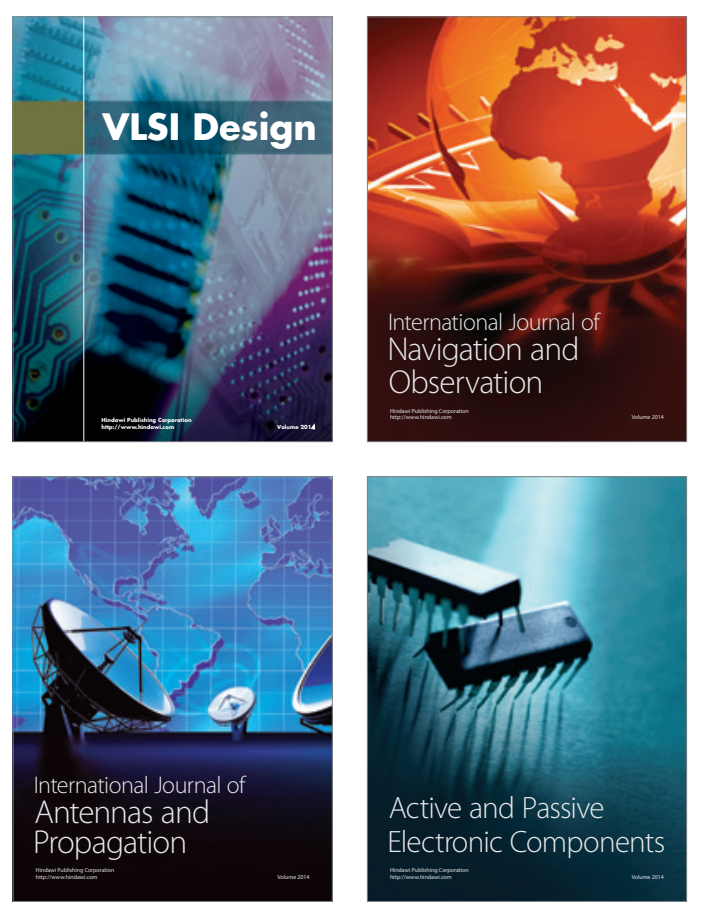
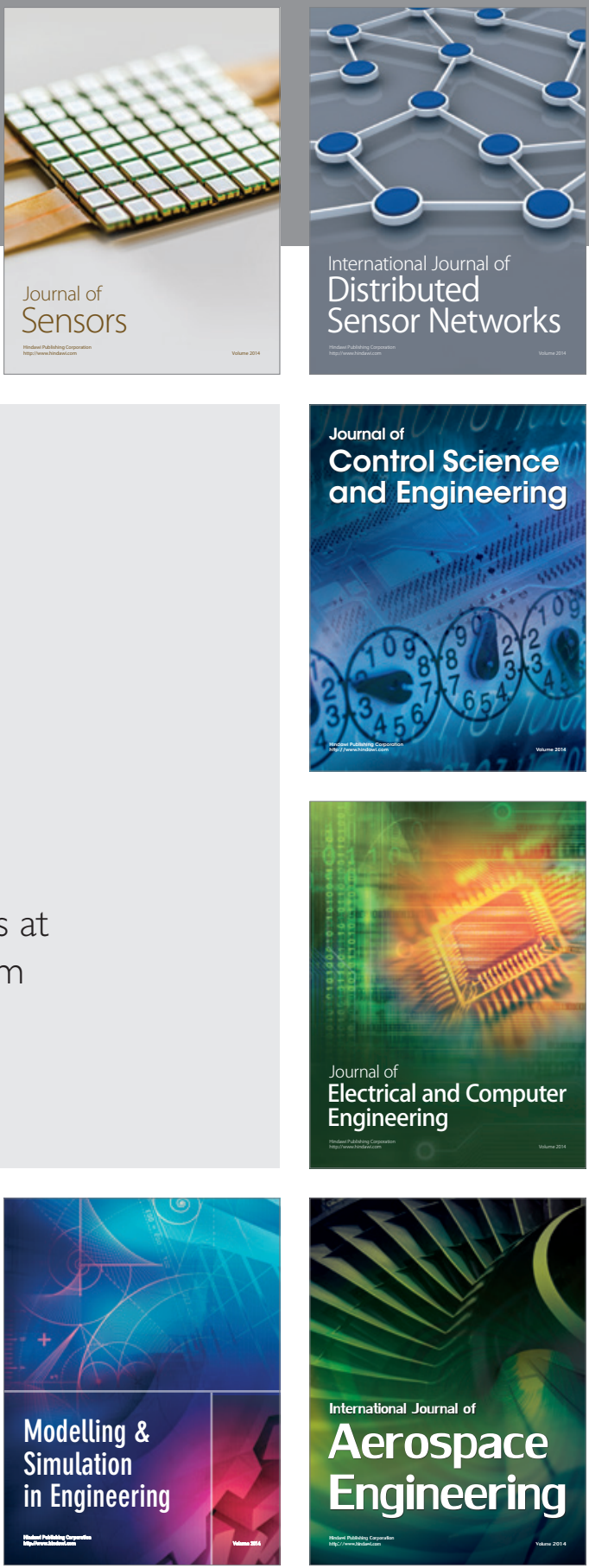

Journal of

Control Science

and Engineering
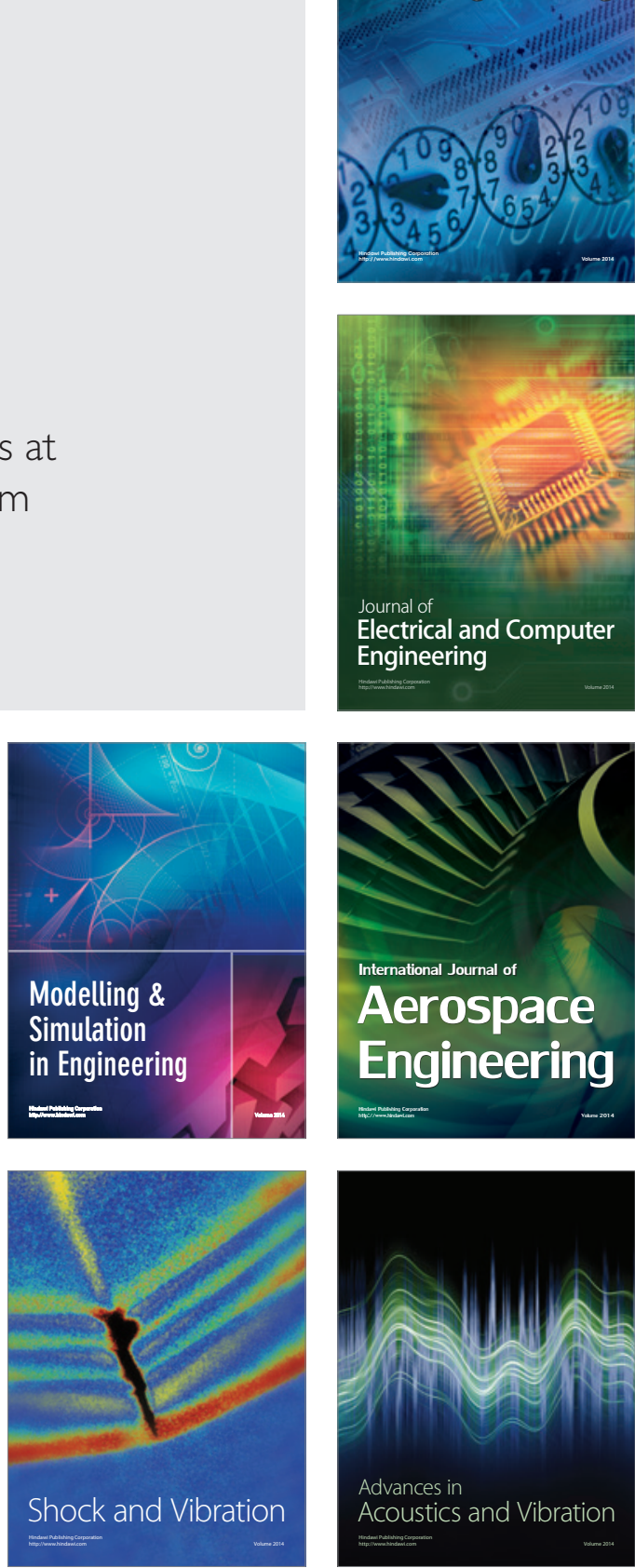